\title{
In-situ pore-scale imaging and image-based modelling of capillary trapping for geological storage of $\mathrm{CO}_{2}$
}

\author{
P.E. Øren ${ }^{\mathrm{a}, \mathrm{b}, *}$, L.C. Ruspini ${ }^{\mathrm{a}}$, M. Saadatfar ${ }^{\mathrm{b}}$, R.M. Sok ${ }^{\mathrm{b}}$, M. Knackstedt ${ }^{\mathrm{b}}$, A. Herring ${ }^{\mathrm{b}}$ \\ ${ }^{\text {a }}$ Petricore Norway AS, Trondheim, Norway \\ ${ }^{\mathrm{b}}$ Australian National University, Canberra, Australia
}

\section{A R T I C L E I N F O}

\section{Keywords:}

Capillary trapping

$\mathrm{CO}_{2}$ storage

Micro-CT

Imaging

Pore-scale modelling

\begin{abstract}
A B S T R A C T
We describe an imaging and pore-scale modelling study of capillary trapping in the Paaratte Sandstone formation in the Otway Basin, Australia. Three-dimensional X-ray computed tomography (micro-CT) was used to characterize the pore structure of the reservoir core. We obtain in-situ pore-scale images of the distribution of $\mathrm{CO}_{2}$ :brine analogue fluid pairs (octane:brine) within reservoir samples during low capillary number drainage and imbibition flooding experiments. The images were recorded using time-lapse X-ray micro-tomography at elevated pressure. The observed two-phase fluid distributions are consistent with a water wet system. The microCT images are used directly as input to a geometrically accurate quasi-static pore-scale simulation model. The validity of the quasi-static assumption is investigated by comparing on a pore-by-pore basis the simulated and imaged fluid distributions. The pore filling states are in good agreement both for drainage and imbibition displacements and the computed capillary trapping curve agrees with experimental data. This indicate that quasistatic pore-scale physics can be used to obtain averaged or continuum flow properties for low capillary number displacements. We perform a sensitivity study of the impact of the advancing contact angle on capillary trapping. The magnitude of residual trapping increases with decreasing contact angle. Land's trapping coefficient increases with increasing contact angle. We compute capillary pressure and relative permeability scanning curves. Simulated relative permeability hysteresis is compared with that predicted by the industry-standard Carlson's and Killough's models. Killough's model reproduces the simulated data more accurately.
\end{abstract}

\section{Introduction}

The adoption of carbon capture and storage (CCS) depends on the effectiveness of immobilising injected $\mathrm{CO}_{2}$ at a geological storage site. The main mechanisms that contribute to immobilise $\mathrm{CO}_{2}$ are; stratigraphic and structural trapping; solubility; mineralisation; and capillary or residual trapping. These mechanisms occur on different time scales without much temporal overlap. Capillary trapping, where $\mathrm{CO}_{2}$ is immobilised by fluid and surface forces as disconnected blobs in the pore space, is a fast trapping mechanism that occurs when brine invades the pore space after the injection of $\mathrm{CO}_{2}$. Recent research has demonstrated that capillary trapping of $\mathrm{CO}_{2}$ is a key mechanism that can immobilise a significant fraction of the injected $\mathrm{CO}_{2}$ in sandstones (Krevor et al., 2015; Burnside and Naylor, 2014; Herring et al., 2016). Capillary or residual trapping is pervasive over large distances of the plume extent and plays a significant role in storage security, slowing plume migration and increasing storage capacity. Small scale heterogeneities and variations in wettability strongly affect the amount of capillary trapping and represent significant uncertainties in storage predictions.

Capillary trapping of the non-wetting phase ( $n w p)$ affects strongly how multiple phases flow in porous media and is of great importance in several practical applications, including soil physics, aquifer remediation, and hydrocarbon recovery methods. In these last processes the aim is to minimise the amount of capillary trapping through manipulation of fluid and flow properties. In contrast, in CCS the goal is to maximise the magnitude of trapped $\mathrm{CO}_{2}$ through several trapping mechanisms, including capillary trapping. The amount of capillary trapping for a given rock depends on the balance between viscous and capillary forces, wettability (i.e. contact angle), and the initial saturation and topology of the injected non-wetting phase.

For a water wet system, the residual saturation increases as the initial nwp saturation increases. The constitutive relation between initial and residual nwp saturations is known as the IR (initial-residual) curve. Several empirical trapping models have been developed to describe the IR relation. The most widely used model for water wet systems is that of Land (1968). In this model the residual saturation, $S_{n w r}$,

\footnotetext{
* Corresponding author.

E-mail address: paal.eric.oeren@petricore.com (P.E. Øren).
} 
is calculated as

$S_{\mathrm{nwr}}=\frac{S_{\mathrm{nwi}}}{1+\mathrm{CS}_{\mathrm{nwi}}}$

where $C$ is Land's coefficient and $S_{n w i}$ is the initial nwp saturation. According to Land's model, the residual saturation increases with the initial saturation, but with a decreasing slope at higher $S_{n w i}$.

Recent work has demonstrated that the $\mathrm{CO}_{2}$ :brine capillary trapping curve for Berea sandstone is invariant across a wide range of pressures and temperatures and that it is the same as that for analogue fluid pairs under water wet conditions (Niu et al., 2015; Krevor et al., 2012). This suggests that for capillary dominated flow in water wet rocks, the IR characteristics is an intrinsic rock property that is controlled by the geometrical and topological properties of the pore structure. Like other constitutive relations describing multiphase flow, the capillary trapping curve is assumed to be invariant to system parameters such as temperature, pressure, interfacial tension, viscosity and salinity. However, the IR characteristics will be different if the system parameters alter the wetting state of the rock or if they change significantly the balance between viscous and capillary forces.

The balance between viscous and capillary forces is described by the dimensionless capillary number $\mathrm{Ca}$. In the literature, $\mathrm{Ca}$ has several definitions depending on the intended application (Hilfer and Øren, 1996; Hilfer et al., 2015). Typically, it is defined in terms of a pore-scale force balance

$\mathrm{Ca}=\frac{v_{i} \mu_{i}}{\sigma}$

where $v_{i}$ is the velocity of the invading phase $i, \mu_{i}$ is the viscosity, and $\sigma$ is the interfacial tension. For water wet rocks, the amount of capillary trapping is constant at small values of $\mathrm{Ca}$ but decreases sharply when $\mathrm{Ca}$ exceeds a critical value. For Berea sandstone the critical value is observed to be $\mathrm{Ca}>10^{-5}$ (Taber, 1969; Youssef et al., 2015; Khishvand et al., 2016). For a wide variety of natural rocks, the critical capillary number is found to be in the range $\mathrm{Ca}>10^{-7}-10^{-4}$ (Lake, 1989). This is orders of magnitude larger than that encountered in $\mathrm{CO}_{2}$ storage situations where the natural flow of brine may only be a few meters per year (Krevor et al., 2015).

Capillary trapping depends strongly on wettability (i.e. the contact angle between the $n w p$ and the solid surface). It is generally expected that a $\mathrm{CO}_{2}$-brine-rock system will be water wet. This is heavily supported by core-flooding experiments (Akbarabadi and Piri, 2013; Krevor et al., 2012; Pentland et al., 2011; Zuo and Benson, 2014; Niu et al., 2015) and by in-situ contact angle measurements using X-ray computed tomography (Andrew et al., 2014a). The wettability or contact angle for a given system is governed by rock-fluid and fluid-fluid interactions. It varies with parameters such as mineralogy and surface roughness in ways that are difficult to estimate. The impact of contact angle variation on the amount of capillary trapping depends strongly on the rock pore structure (Chatzis and Morrow, 1984; Suzanne et al., 2003; Ruspini et al., 2017).

Recent advances in X-ray micro-tomography and 3D image registration allows direct visualisation of fluid distributions and $\mathrm{CO}_{2}$ trapping processes at the pore-scale as well as quantitative measurements of in-situ contact angles at conditions representative of the storage reservoir (Andrew et al., 2014a; Blunt et al., 2013). The micro-CT images and the measured in-situ contact angles can be used directly as input to pore-scale simulators. The imaged pore-scale fluid distributions can be used to calibrate and investigate the predictive capabilities of these simulators on a pore-by-pore basis (Bultreys et al., 2018). For capillary dominated flow, the amount and distribution of trapped nwp are determined by the balance between the two bond-breaking mechanisms: snap-off and cooperative pore body filling (Lenormand et al., 1983). This, in turn, depends on geometrical and topological characteristics of the pore structure, such as aspect ratio and coordination number (Ruspini et al., 2017).
In this work we describe an imaging and pore-scale modelling study of capillary trapping in the Paaratte Sandstone formation in the Otway Basin, Australia. We obtained in-situ pore-scale images of the distribution of $\mathrm{CO}_{2}$ :brine analogue fluid pair (octane:brine) within reservoir samples during low capillary number drainage and imbibition experiments. The 3D pore structure images are used directly as input to a new geometrically accurate quasi-static pore-scale simulation model. We simulate nwp injection (drainage) and subsequent brine imbibition and examine the predictive capabilities of the model by comparing on a pore-by-pore basis the calculated fluid distributions with the corresponding micro-CT imaged distributions. We compute capillary trapping curves and perform a sensitivity study of the impact of wettability (i.e. contact angle) on the magnitude of capillary trapping. Finally, we simulate capillary pressure scanning curves and the corresponding hysteretic relative permeability behaviour.

\section{Materials and methods}

\subsection{Experimental}

The experiments were designed with the goal of understanding and accurately quantifying the pore-scale distribution of fluids within the reservoir sample during drainage and imbibition displacements. Low capillary number $\left(C a \simeq 10^{-9}\right)$ octane injection (drainage) and brine injection (imbibition) experiments were conducted in a high-pressure flow cell designed for implementation within a laboratory-based X-ray CT (micro-CT) tomographic system.

A high pressure, high temperature triaxial flow cell (HPTC) was designed using an aviation grade aluminum alloy (T7000 series) capable of withstanding radial and axial pressures of up to 10,000 psi (see Fig. 1). The HPTC is designed to accommodate cores of up to $12 \mathrm{~mm}$ in diameter and $40 \mathrm{~mm}$ in length and can be mounted on micro-CT machines for in-situ experiments (see Fig. 1b and c). This experimental run was conducted under moderate pressure (specific values in Table 1) and ambient temperature conditions.

There are four pumps in the system: two hydraulic oil pumps (Metarock Laboratories, Houston, TX, United States) controlling confining and axial fluid pressure, and two Hastelloy pumps (Teledyne ISCO, Lincoln, NE, United States) controlling injection fluids.

Samples were cored and cut to $12 \mathrm{~mm}$ diameter and $36 \mathrm{~mm}$ length; the samples were bounded on both ends by viton o-rings and copper sintered flow diffusers and incased in shrink-wrap which acts a barrier between confining pressure fluid and the experimental working fluids. The cores were installed inside the HPTC core holder, and axial pressure was applied by tightening the upper end cap until vertical displacement (of approx. $0.001 \mathrm{~mm}$ ) of the core was measured via an internal Linear Variable Differential Transformer (LVDT) (Solartron Metrology, USA). At this point, axial pressure was applied via pumping hydraulic fluid to an axial pressure of approx. $100 \mathrm{psi}$, after which confining fluid was pumped into the radial region between the shrinkwrap and the core holder walls and elevated to 50 psi. Axial and radial confining pressure were then elevated simultaneously to working pressures of 350 and $300 \mathrm{psi}$, respectively.

Axial stress must be applied in order to compress the o-ring at each axial face of the core and prevent cross-flow of confining oil and working fluids. Application of radial confining pressure exerts some vertical force on the core and could potentially cause slippage of the oring. Additional axial confining pressure must therefore be applied in order to accurately maintain the vertical position of the core and prevent o-ring slippage. This necessitates simultaneous elevation of both radial and axial pressures during pressurisation. The sandstone cores used in this study are fragile and it was necessary to use this protocol to prevent damaging the cores (in contrast to simply over-tightening the mechanical screw thread to apply a high axial pressure at the start of the experiment).

Experimental data sets during each experimental run were acquired 

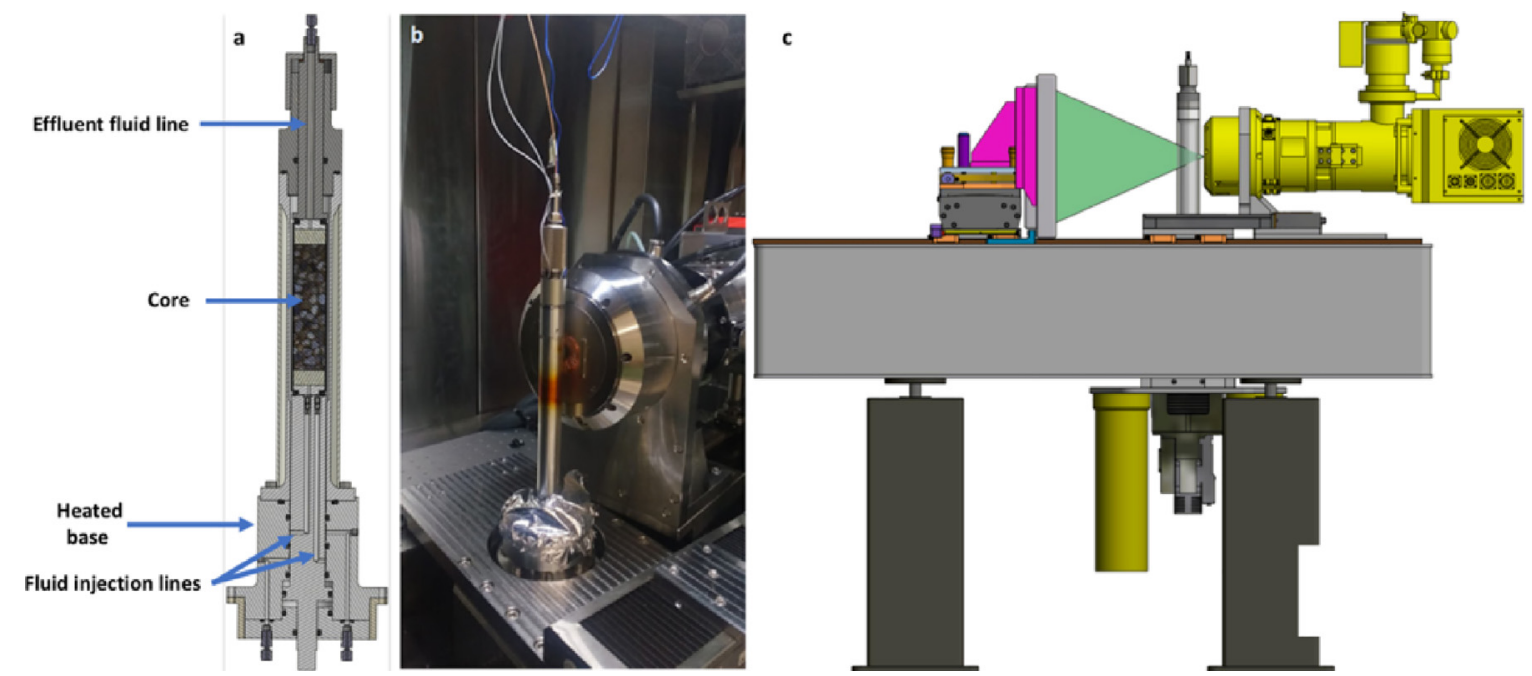

Fig. 1. (a) CAD drawing of HPTC showing the rock core and flow inlets/outlet as well as hydraulic fluid connections. (b) The HPTC can be mounted on the rotational stage of the micro-CT for in-situ imaging. (c) CAD drawings depicting the HPTC and the micro-CT system.

in a dry state, after brine saturation, after octane drainage and after brine imbibition. The "dry" scan was acquired after axial and confining pressure was applied, prior to any fluid injection. After dry scan acquisition, the core was saturated with $0.5 \mathrm{M}$ KI brine at relatively high flow rate (i.e. the fastest flow rate that maintained brine pore pressure below $300 \mathrm{psi}$, as reported by the ISCO internal pressure transducer). Specific information on flow rates/working pressures are given in Table 1 . Then, the pore pressure was incrementally increased to the desired working pore pressure via the back-pressure regulator, while still flowing brine, to attempt to simultaneously dissolve any remaining air bubbles and flush them from the core. Some air bubbles were still present after this process; however, regions were present that represented nearly $100 \%$ brine saturation. Pore pressure was maintained via the brine pump while the second scan was acquired of this brine saturated state.

Drainage was carried out by injecting octane at constant flow rate for a total of 10 estimated pore volumes; after which pore pressure was maintained via the pump for the duration of the imaging of this drained state. Finally, brine was imbibed at constant flow rate for 10 pore volumes to reach "residual" saturation. The pore pressure was maintained via the brine pump while the imbibition scan was acquired. Radiographs were acquired during each flow process to monitor the extent of flow and to check that a steady state was achieved (i.e. no changes were observed over dozens of consecutive radiographs spanning approx. $30 \mathrm{~min}$ ) before tomographic imaging was initiated. Flow was halted during scan acquisition. Short-term (i.e. immediately after flow cessation) capillary redistribution was not monitored by radiographs. However, no major movement artifacts were observed in the tomograms, suggesting that quasi-static equilibrium is achieved during imaging. All scans were acquired for approx. 20-24h and were collected as region of interest (ROI) scans using a double helix acquisition pattern.

\subsection{Pore network model generation and flow modelling}

Multiphase flow in the micro-CT images are simulated using a geometrically accurate pore network model. The dry micro-CT images are transformed into a topologically equivalent pore network that retains the essential features of the pore space that are relevant to fluid flow. We applied a new pore-based algorithm for extracting network representations of micro-CT images. The new algorithm involves five basic steps:

1. A Euclidean distance transformation algorithm (Saito and Torimaki, 1994) is first applied to the pore space.

2. The skeleton of the pore space is generated following a similar approach to that described in Yi et al. (2017). The pore-based skeleton is extracted using a medial surface axis thinning algorithm (Lee et al., 1994) that ensures the in-variance of the Euler characteristics between the image and the skeleton. In addition to the Euler invariance, a set of rules for simple points and connectivity are used to preserve the topological properties of the pore space.

3. The pore-based skeleton is next populated with maximum balls and the network structure is defined following the methodology described in Silin and Patzek (2003) and Dong and Blunt (2009). All the maximum balls are sorted according to size and the ancestors are defined as the larger balls in the neighbourhood defined by the overlapping local balls. The ancestors balls are the nodes. The links are defined by the balls that have multiple ancestors.

4. The pore space is fully segmented using a unique label for each of the pore elements (nodes and links). The voxels (i.e. volume) assigned to a pore element is defined by the inscribed radius at the center of the pore. For each link, two planes perpendicular to the skeleton voxels are defined. The plane centers are located in the first free skeleton voxels of the two connecting nodes. The perpendicular direction of these planes is calculated based on a weighted average of the directions of the four neighbouring voxels. The domain of the nodes and links are defined by these planes. This ensures that the pore boundaries are perpendicular to the skeleton.

5. The parameters used to describe the geometrical characteristics of each pore elements (nodes and links) are given in Øren et al. (1998) and Øren and Bakke (2003). In this work, a new method for measuring the geometrical parameters is used. The geometrical properties assigned to nodes are the average of the values measured on

Table 1

Fluid flow and CT acquisition parameters for flooding experiments. FOV denotes field of view.

\begin{tabular}{llllllll}
\hline Drainage flow rate & Drainage volume & Imbibition flow rate & Imbibition volume & Pore pressure & X-ray voltage & Current & Vertical FOV \\
\hline $0.18 \mathrm{ml} / \mathrm{min}$ & $10 \mathrm{PV} ; 12 \mathrm{ml}$ & $1.8 \mathrm{ml} / \mathrm{min}$ & $10 \mathrm{PV} ; 12 \mathrm{ml}$ & $150 \mathrm{psi}$ & $120 \mathrm{keV}$ & $60 \mathrm{~mA}$ & $19.7 \mathrm{~mm}$ \\
\hline
\end{tabular}


the cross-sectional planes perpendicular to the direction of the connecting links. Similarly, the geometrical properties of the links are measured on the cross-sectional plane at the narrowest constriction of the link in the direction that is perpendicular to the skeleton direction.

All the above-defined algorithms are parallelised in order to increase the performance of the network extraction for large samples.

The pore network simulator used in this work is based on the simulator first presented in Øren et al. (1998). The network simulator accounts for pore-scale wettability, wetting and non-wetting phase layers (van Dijke and Sorbie, 2006) and buoyancy forces. It is extended to include the new way of measuring geometrical properties described above and it incorporates the geometrically accurate model for cooperative pore body filling displacements described in Ruspini et al. (2017). This model accounts the geometrical characteristics of the pore body, the spatial location of the connecting throats, and the local fluid configuration at the time of the pore filling displacement. It has been validated against experimental data and describes correctly the dependency of capillary trapping on contact angles, connectivity and aspect ratio that previous models failed to reproduce (Ruspini et al., 2017).

Fluid displacements in the pore network are modeled using the assumption of quasi-static pore-scale physics: the displacement is so slow that viscous forces can be neglected and only capillary and buoyancy forces need to be accounted for. At each moment the fluids are assumed to be at capillary equilibrium. Predicting the evolution of the displacement therefore reduces to calculating the entry capillary pressure associated with available pore elements and invade elements in order of this entry pressure (Lenormand et al., 1983; Blunt et al., 1992; Øren et al., 1998). Capillary entry pressures depends on the local pore geometry, wettability and initial fluid configuration (Mayer and Stowe, 1965; Princen, 1969; Kovscek and Radke, 1993; Øren et al., 1998; Piri and Blunt, 2004; van Dijke et al., 2004, 2007; Ruspini et al., 2017).

\section{Results and discussion}

A plug sample from the Paaratte Sandstone formation in the Otway Basin, Australia, is analysed. The sample is classified as part of the massive sand reservoir facies - a fine to medium grained, sub angular to rounded well sorted sandstone with mainly quartoze grains. The plug is from the interval between 1535 and $1537 \mathrm{~m}$ depth. As described in the experimental section, low capillary number $\left(\mathrm{Ca} \simeq 10^{-9}\right)$ octane drainage and brine imbibition micro-CT flooding experiments were conducted. The octane:brine interfacial tension was approx. $35 \mathrm{mN} / \mathrm{m}$ and the brine and octane densities were 1123 and $703 \mathrm{~kg} / \mathrm{m}^{3}$, respectively.

Four 3D scans were acquired in dry, saturated, drained and imbibed states. Radiographs were acquired during each flow process to ensure that steady state was achieved (i.e. no changes were observed over dozens of consecutive radiographs spanning approx. $30 \mathrm{~min}$ ) before imaging was initiated. The images were segmented into three phases; solid; intermediate/clay phase; and pore using the three-phase converging active contours segmentation method (Sheppard et al., 2004). The intermediate/clay phase was set to a partial porosity of $50 \%$. Table 2 summarizes the properties of the segmented dry micro-CT image. The voxel resolution is $6.07 \mu \mathrm{m}$ and the total porosity, $\phi_{t o t}$, is 0.27 . The image contains a small amount of clay $\left(f_{\mu} \simeq 3.5 \%\right)$ with subresolution porosity. The vertical permeability $k_{z z}$ and formation factor

\section{Table 2}

Properties of the segmented micro-CT image. $\phi_{r e s}$ is the resolved porosity and $\phi_{\mu}$ is the total porosity of the intermediate/clay phase.

\begin{tabular}{lllllll}
\hline Size [voxels] & $a[\mu \mathrm{m}]$ & $\phi_{\text {res }}[\%]$ & $f_{\mu}[\%]$ & $\phi_{\mu}[\%]$ & $k_{z z}[\mathrm{mD}]$ & $F_{z z}$ \\
\hline $792 \times 764 \times 1180$ & 6.077 & 25.29 & 3.56 & 1.75 & 2796 & 14.0 \\
\hline
\end{tabular}

$F_{z z}$ calculated directly on the dry image are also given in Table 2 .

$2 \mathrm{D}$ slices through the $3 \mathrm{D}$ micro-CT data at the various saturation states are shown in Fig. 2. The observed pore-scale fluid distributions are consistent with a water wet system. Octane is the non-wetting phase and preferentially invades the larger pore spaces. Brine is the wetting phase and occupies the smaller pores and corners and crevices of pores invaded by octane. After imbibition, the residual $n w p$ is trapped as isolated blobs or ganglia of all sizes, from single pore ganglia to multipore ganglia that nearly span the system, consistent with a water wet system.

A pore network model was extracted using the porosity map image obtained from the difference between the dry and fully saturated microCT scans, where resolved and unresolved void regions can be characterised. Geometrical and petrophysical properties of the extracted pore network are listed in Table 3. The sample is well connected with an average coordination number $\bar{Z}$ (no. of links connected to a node) of 5.05. The average aspect or contraction ratio $\overline{\mathrm{AR}}$ (pore body radius to pore throat radius) is small (1.73) compared to what it is typically observed for consolidated rocks (Ruspini et al., 2017). In water wet porous media, the aspect ratio controls the balance between snap-off in throats and piston type invasion in pore bodies. This has important effects on the displacement behavior and the magnitude of capillary trapping during brine injection. The network permeability and formation factor are similar to that calculated directly on the micro-CT image. The pore-size distributions for nodes (pore body) and links (throats) are displayed in Fig. 3. The sample is relatively homogeneous with a narrow range of pore and throat sizes. The average link size is $\overline{r_{l}} \simeq 12.5 \mu \mathrm{m}$ and the average node size is $\overline{r_{n}} \simeq 19.5 \mu \mathrm{m}$.

We simulate octane injection (drainage) and subsequent brine injection (imbibition) in the pore network. Octane is injected at the inlet face (bottom) of the fully saturated network and brine escapes through the outlet on the opposite side (top), mimicking the experimental boundary conditions. The simulation stops when the octane saturation reaches a target value, $S_{g i}$. We assume water wet conditions for all the simulations, in agreement with the experimental observations. For primary drainage, the receding contact angles are randomly distributed in the range $\left[0-10^{\circ}\right]$. We use advancing contact angles randomly distributed in the range $\left[30-40^{\circ}\right]$ as the base case for imbibition.

\subsection{Simulated vs. experimental fluid distributions}

Recent observations of dynamic effects in micro-CT flooding experiments challenge the assumption that quasi-static pore-scale physics is valid at low capillary numbers, $\mathrm{Ca}<10^{-6}$, (Wildenshild and Sheppard, 2013; Berg et al., 2013; Schlüter et al., 2017). However, it is not known how, or if, the quasi-static approximation deviates from experimental reality. We investigate this by comparing the simulated pore-scale fluid distributions after primary drainage and imbibition with the corresponding experimental fluid distributions. The primary drainage simulation is terminated at the same global saturation as in the experiment, $S_{w i}=0.52$. The simulated pore-scale fluid distributions are mapped back to the micro-CT image to allow consistent and direct comparison to the experiments. This saturation mapping is possible since the network model is extracted directly from the micro-CT image and it stores which voxels belong to each pore element. Fig. 4 compares the experimental and simulated fluid distributions after primary drainage in the central slice perpendicular to the injection direction. Visually, the distributions are clearly similar. The nwp (octane) preferentially occupies the larger pores in both images, in agreement with the water wet state of the system.

We analyse statistically the differences in pore filling states between the experimental and simulated distributions by plotting pore occupancy as a function of pore radius. The radius of a pore element is defined by the inscribed sphere at the centre of the element. These inscribed spheres are used to assign the filling state of a pore element. A pore or throat is said to be occupied by non-wetting phase if the voxel at 


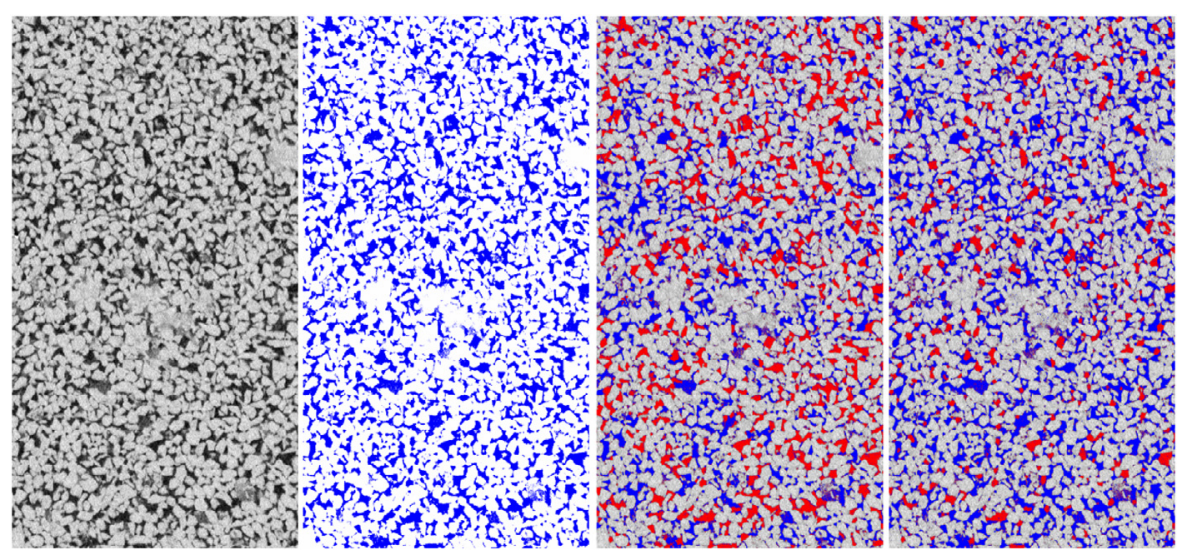

Fig. 2. 2D slices through the 3D tomographic data of sample DC8. From left to right: dry image; Brine saturated ( $S_{w} \simeq 1$ ), brine regions (blue), solid regions (white) and clay (gray); after octane (red) drainage $\left(S_{w}=0.52\right)$; after brine flooding $\left(S_{w}=0.78\right.$ ), phase distributions at residual $n w p$ saturation. (For interpretation of the references to color in this figure legend, the reader is referred to the web version of this article.)

Table 3

Geometrical and petrophysical properties of the extracted pore network.

\begin{tabular}{lllllllll}
\hline Nodes & Links & $\phi_{\text {tot }}$ & $\overline{\mathrm{AR}}$ & $\bar{Z}$ & $\overline{r_{l}}[\mu \mathrm{m}]$ & $\overline{r_{n}}[\mu \mathrm{m}]$ & $k_{z z}[\mathrm{mD}]$ & $F_{z z}$ \\
\hline 192,662 & \multirow{2}{*}{399,482} & 27.04 & 1.73 & 5.05 & 12.46 & 19.49 & 2542 & 14.8 \\
\hline
\end{tabular}

the centre of the inscribed sphere contains nwp and if more than half of the voxels associated with the inscribed sphere contains non-wetting phase.

Fig. 5 compares the simulated and experimental pore occupancy after primary drainage. The brine filled pore distributions are shown in blue (experimental) and green (simulated). As expected for a water wet system, brine occupies the smallest pores. The nwp (octane) filled pore distributions are shown red (experimental) and violet (simulated). Overall, we observe a strong correlation of pore occupancy as a function of pore size for the two systems. The filling discrepancy as a function of pore size is shown in yellow. The filling discrepancy is largest for the smallest nodes and links. For nodes, the filling discrepancy is $6.8 \%$ of the total nodes while for the links the discrepancy increases to $10.1 \%$, mainly due to different filling states in the smallest links. However, in terms of saturation the total (nodes and links) filling discrepancy is less than $3 \%$.

In the experimental link distribution, a peak of $n w p$ filled links is observed at small radii $\left(r<10^{-5} \mathrm{~m}\right)$. The capillary entry pressures for these links are high and unlikely to be reached during the low capillary number drainage conditions considered here. This suggests that the filling discrepancy for the small links is due mainly to segmentation errors (i.e. noise) or a small misalignment when subtracting different images. Due to the finite resolution of the images it is hard to accurately segment the fluid distribution in these small links that are roughly the size of the voxel resolution.

The same statistical pore filling analysis is done for the imbibition displacements. To avoid the simulation errors from the primary drainage, we have mapped the experimental fluid distribution into the network model and used it as the initial state for the imbibition simulation. The simulation stops when the residual octane saturation is reached (i.e. when octane is no longer connected across the network). The simulated and experimental residual saturations were similar $\left(S_{o r} \simeq 0.22\right)$.

Fig. 6 shows a comparison between the imaged and simulated fluid distributions after imbibition. A relatively good match is observed, especially considering the complex competition between different porescale imbibition displacement mechanisms (Ruspini et al., 2017) and the difference between experimental and simulated boundary conditions (due the fact that the image only represent the central part of the plug). Despite of these uncertainties, a significant part of the pore elements has the same fluids occupancy in both systems. This is shown in

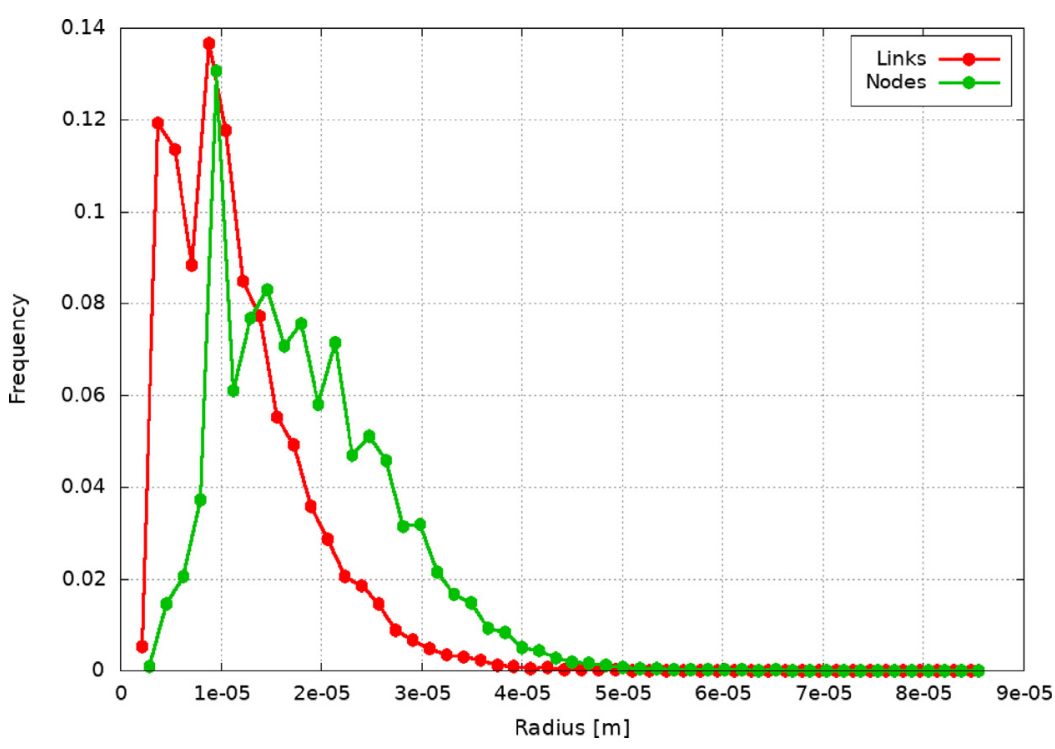

Fig. 3. Pore size distribution for nodes and links in the pore network extracted from the micro-CT images. 


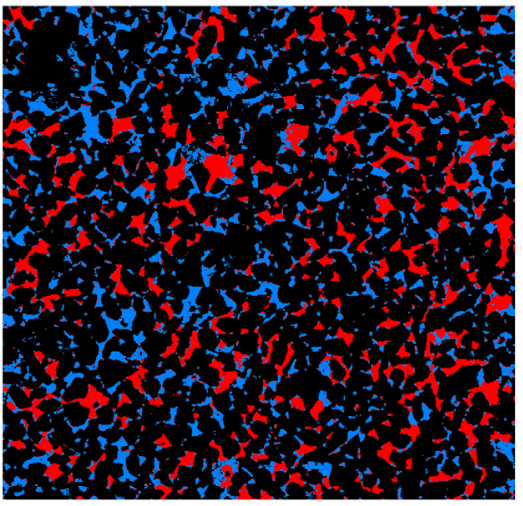

(a)

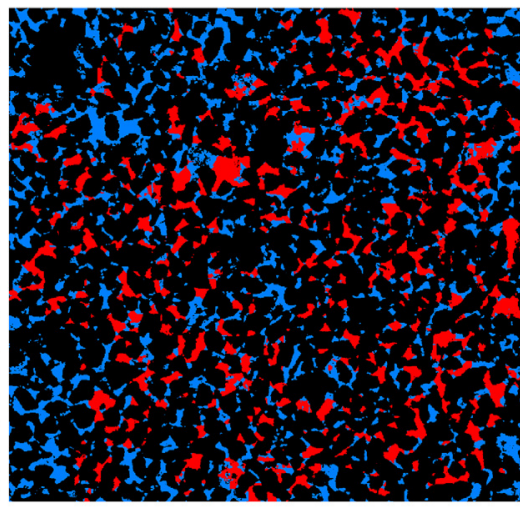

(b)

Fig. 4. Experimental (left) and simulated (right) fluid distributions after primary drainage, $S_{w}=0.52$. Octane is red, brine is blue, and solid is black. (For interpretation of the references to color in this figure legend, the reader is referred to the web version of this article.)

the filling discrepancy analysis presented in Fig. 7. Discrepancies of $15 \%$ and $13 \%$ are observed for the filling state of nodes and links, respectively. For nodes, the filling discrepancy is largest for intermediate size pores while for links the discrepancy is largest for the smaller throats. The total pore filling discrepancy in terms of saturation is less than $7 \%$.

The observed variability comes from different sources. Two-phase flow is very sensitive to perturbations in the boundary conditions (Ferrari et al., 2015; Ling et al., 2017). The differences between the experimental and simulated boundary conditions (i.e. image only represent the central part of the plug) can trigger differences in the displacement events. Also, small differences in the initial saturation distribution for imbibition may lead to discrepancy in the observed pore filling states. Finally, the experimental pore-scale fluid distributions are not fully reproducible (Menke et al., 2016; Song and Kovscek, 2016). Bultreys et al. (2018) report a discrepancy in the experimental pore filling states of about $10 \%$ for five repeat $\mathrm{CO}_{2}$ :brine micro-CT core flooding experiments in Bentheimer sandstone (Andrew et al., 2013, 2014b).

\subsection{Capillary trapping}

We simulated a total of nine primary drainage and imbibition sequences. We assume strongly water wet conditions for all primary drainage simulations, randomly assigning receding contact angles in the range $\left[0-10^{\circ}\right]$. The simulation stops when the $\mathrm{CO}_{2}$ saturation reaches a target value, $S_{g i}$. Next, we simulate imbibition by injecting brine. The simulation terminates when the $\mathrm{CO}_{2}$ phase is no longer connected to the outlet faces. Fig. 8 shows the simulated initial vs residual (IR) trapping curves for the sample. We performed a sensitivity analysis of the impact of the advancing contact angle on capillary trapping using four sets of advancing contact angles: $\left[10-20^{\circ}\right]$, $\left[20-30^{\circ}\right],\left[30-40^{\circ}\right]$ and $\left[40-50^{\circ}\right]$. The amount of trapping depends on the value of the contact angles and trapping increases with decreasing contact angles. This is due to changes in the competition between snapoff in throats and piston-type displacements in nodes. Capillary entry pressures for snap-off decrease faster with increasing contact angles than do piston-type entry pressures. Snap-off is impeded and pistontype displacements (frontal advance) increase, reducing trapping.

The experimental data point obtained from the micro-CT images is located in the middle of the simulated results, between the $\left[20-30^{\circ}\right]$ and $\left[30-40^{\circ}\right]$ distributions. This agrees with in-situ contact angles found experimentally for sandstones of around $\left[20-50^{\circ}\right]$, as reported in Khishvand et al. (2016). Fig. 8 shows that the capillary trapping curves increases monotonically with the initial saturation until $S_{g i} \simeq 0.6 . S_{g r}$ is nearly constant at higher initial saturations. For large contact angles (i.e. less water wet), the trapped saturation reaches a plateau value and slightly decreases as the initial saturation increases further, similar to that observed in Spiteri et al. (2008).

The best fit Land's coefficient for the base case IR curve is $C=2.5$, as shown in Fig. 9. The relatively high value of $C$ is caused by the combination of high connectivity, low average aspect ratio and narrow

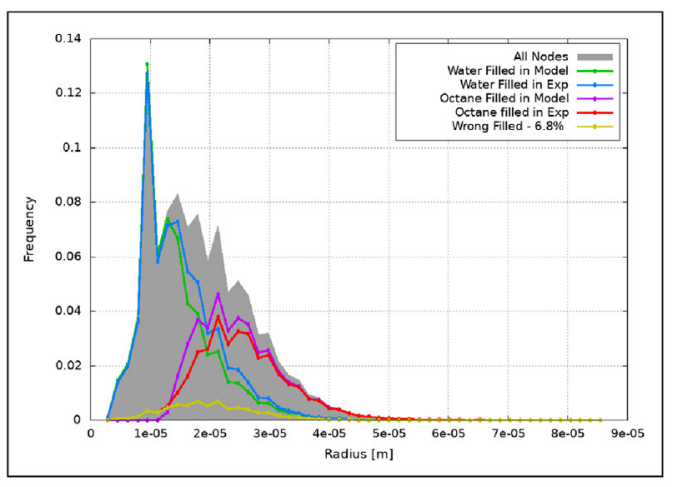

(a)

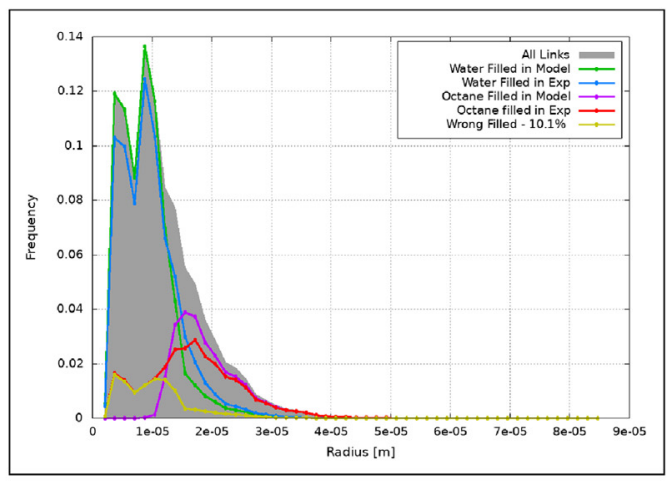

(b)

Fig. 5. Pore fluid occupancy after drainage as a function of pore radius for nodes (left) and links (right). The unit on the $y$-axis is the number frequency normalised to one. 


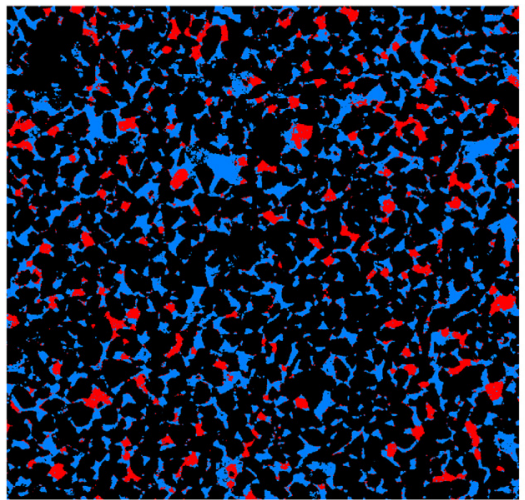

(a)

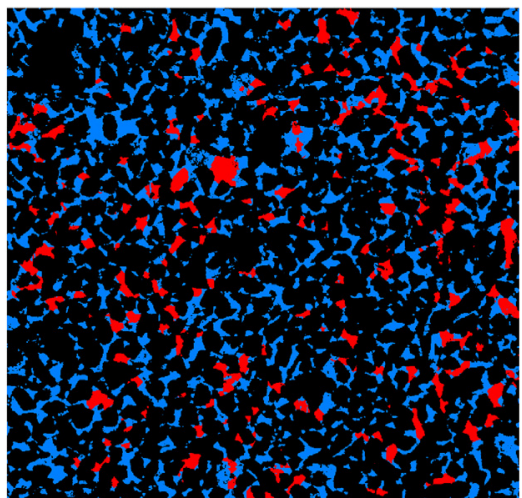

(b)

Fig. 6. Experimental (left) and simulated (right) fluid distributions after imbibition, $\left(S_{w}=0.78\right)$.

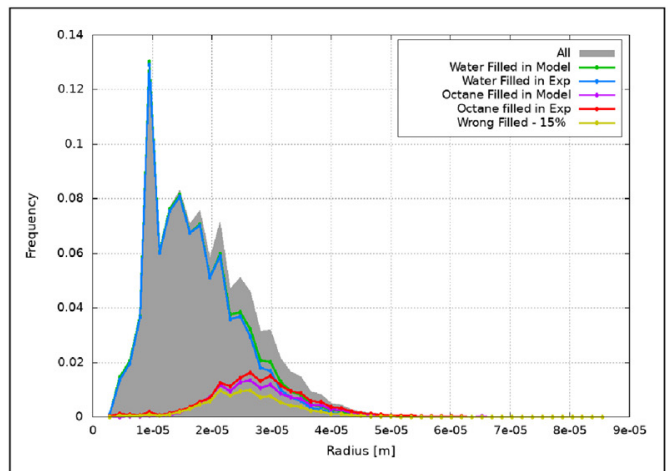

(a)

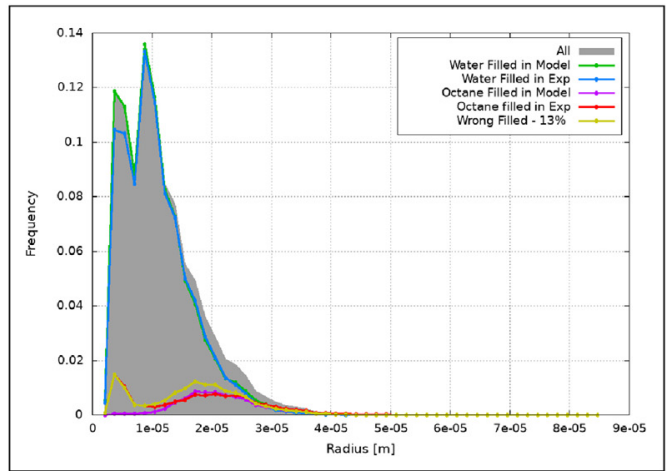

(b)

Fig. 7. Pore fluid occupancy after imbibition as a function of pore radius for nodes (left) and links (right).

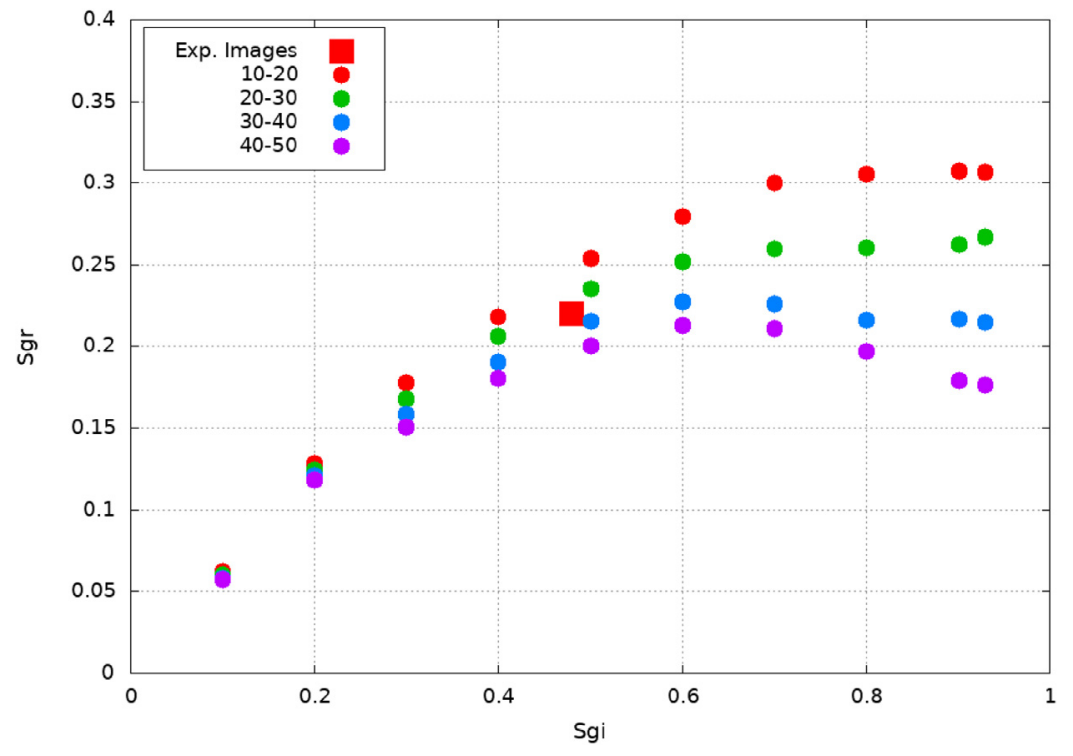

Fig. 8. Capillary trapping curves for four sets of advancing contact angles.

pore size distribution. The low aspect ratio results in a subtle balance between piston-type invasion in pores and snap-off in the connecting throats. If the aspect ratio is small, the entry pressure for piston-type node invasion is greater than that for snap-off. In this case, the wetting phase invades the pore and adjoining throat by piston-like frontal advance and there is little trapping. If the aspect ratio is large, snap-off is the preferred displacement, resulting in trapping of the non-wetting phase in the pore-body.

Also shown in Fig. 9 are simulated (Ruspini et al., 2017) and measured trapping curves for Berea. The simulated data are in good 


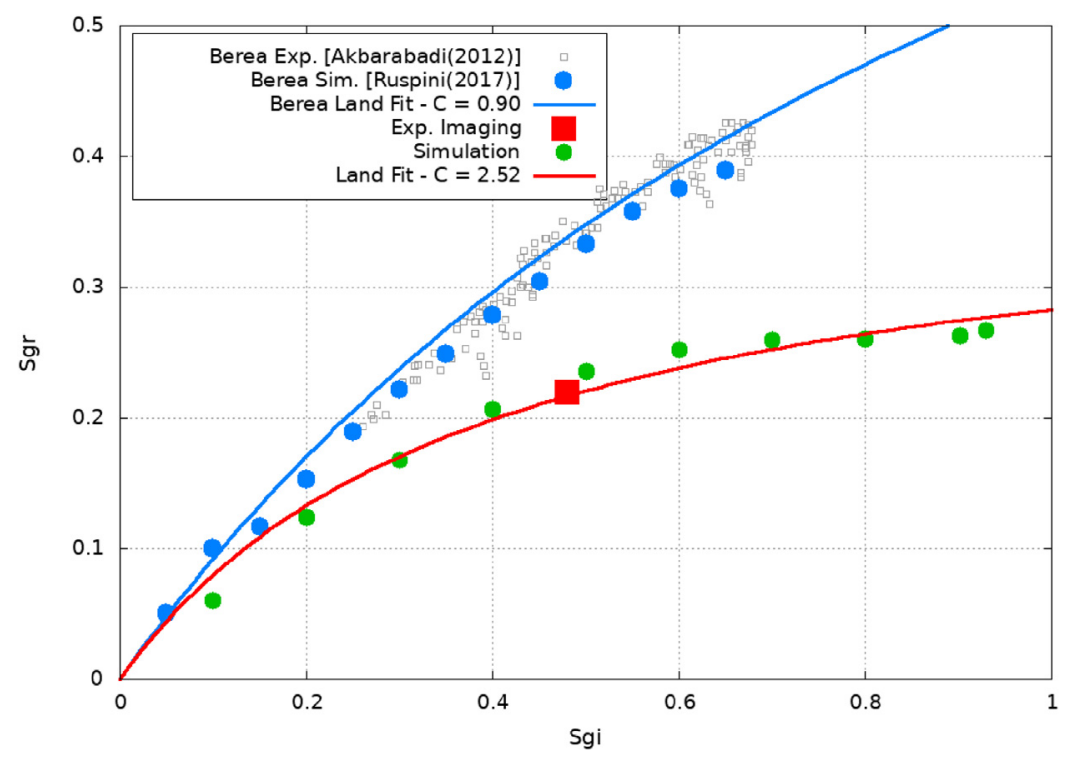

Fig. 9. Land's model fitting and comparison with Berea sandstone data.

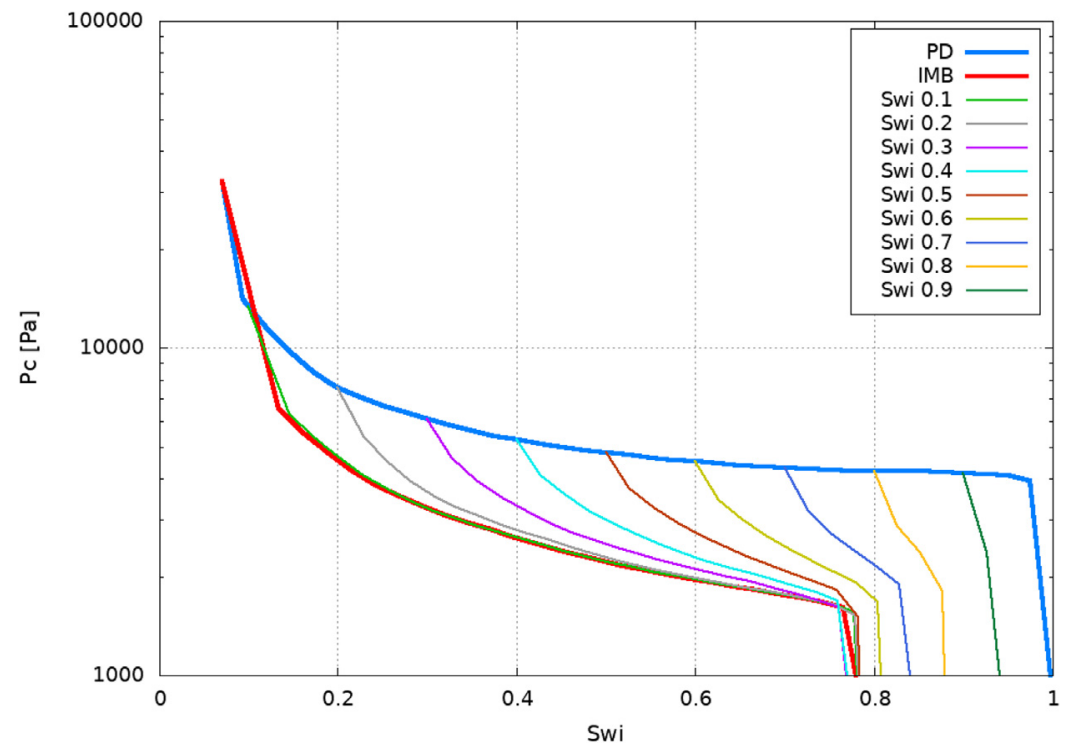

Fig. 10. Capillary pressure scanning curves between the bounding primary drainage (PD) and imbibition (IMB) curves.

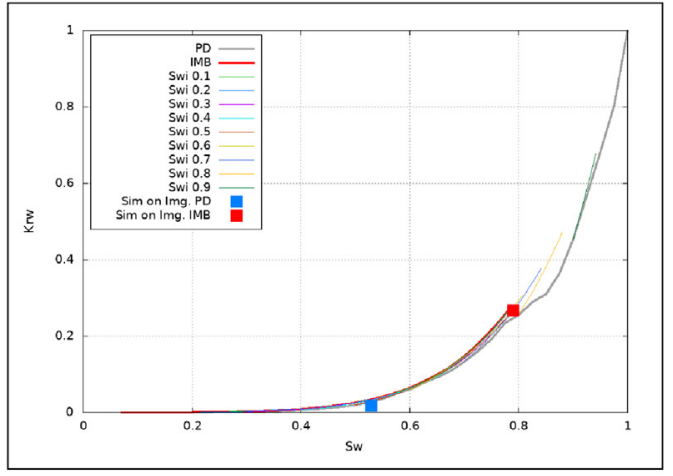

(a)

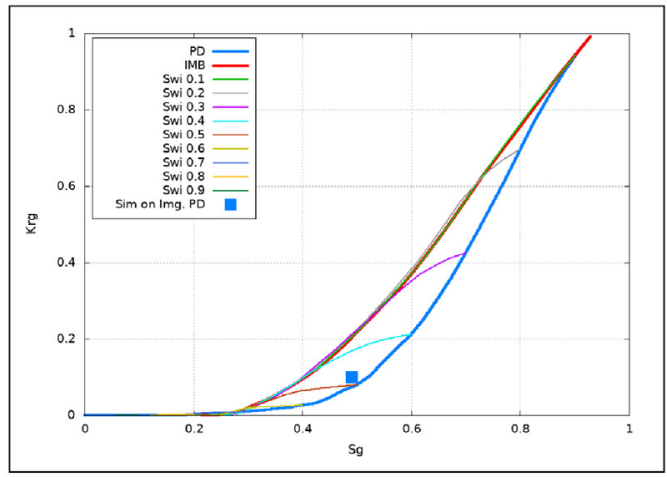

(b)

Fig. 11. Wetting (left) and non-wetting phase (right) relative permeability scanning curves. The blue and red data points are calculated directly on the micro-CT images. 


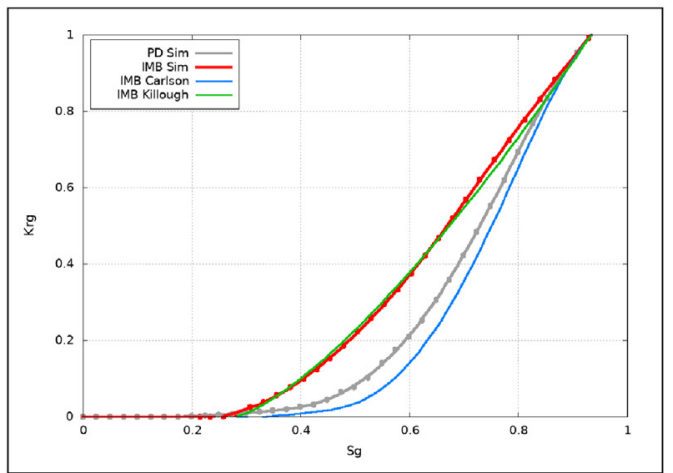

(a)

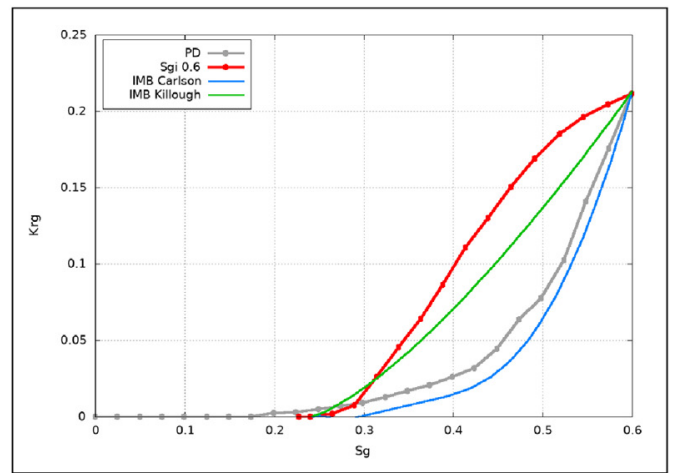

(b)

Fig. 12. Predictions of non-wetting phase imbibition relative permeabilities by the Carlson and Killough hysteresis models compared with the simulated results for $S_{w}=S_{\text {wi }}$ (left) and $S_{w}=0.4$ (right).

agreement with the measured data across most of the initial saturation range and the value of $C=0.9$. This is significantly less than that for the sample analysed in this work, although the porosity and permeability values of the two samples are similar. The different trapping characteristics are caused mainly by the different aspect ratios. The average aspect ratio of Berea is about 2.25 (Øren and Bakke, 2003; Raeini et al., 2015) compared to 1.73 for the sample analysed here. The balance between snap-off and piston-type displacement (i.e. frontal advance) in pores depends strongly on the aspect ratio (Ruspini et al., 2017). Higher aspect ratios result in increased snap-off events and increased capillary trapping (i.e. smaller $C$ values). This analysis highlights the importance of characterising properly the topology and geometry of the pore structure in order to understand and quantify capillary trapping of $\mathrm{CO}_{2}$.

\subsection{Scanning curves and hysteresis analysis}

Fig. 10 shows simulated capillary pressure curves for primary drainage and imbibition displacements commencing at different initial saturations. The drainage capillary pressure curve is L-shaped which reflects the relatively small variation in pore sizes. The scanning capillary pressure curves are as expected for a water wet system. The relative permeability scanning curves are shown in Fig. 11. There is little hysteresis in the brine relative permeability. This is because brine is the wetting phase both during the $\mathrm{CO}_{2}$ and brine injections. However, the non-wetting phase relative permeabilities display clear hysteresis, as shown in Fig. 11(b). Relative permeability points calculated directly on the in-situ micro-CT images using a single phase TRT Lattice Boltzmann solver are in close agreement with the relative permeability curves calculated using the network model.

The $\mathrm{CO}_{2}$ imbibition relative permeability is larger than that for drainage, as shown in Fig. 11(b). The larger non-wetting phase imbibition relative permeability is characteristic of low aspect ratio rocks, as reported by Braun and Holland (1995). Most empirical hysteresis models assume that $k_{r g}$ (imbibition) $<k_{r g}$ (drainage), in accordance with the data reported by Land (1968). In Fig. 12 we compare the bounding $\mathrm{CO}_{2}$ relative permeability curves with the curves obtained using the industry-standard Carlson's (Carlson, 1981) and Killough's (Killough, 1976) models. Carlson's model is designed for lower gas relative permeability during imbibition and the match with the simulated curve is bad. Killough's model uses the bounding imbibition curve (in addition to the drainage curve) to estimate hysteresis in the nwp relative permeability and this model predicts hysteresis curves similar to the simulated results. Fig. 12(b) shows the comparison for an initial nonwetting phase saturations of 0.6. Once more, Killough's model describes better the hysteresis behavior.

\section{Conclusions}

We describe a comprehensive imaging and modelling study of capillary trapping in the Paaratte Sandstone formation in the Otway Basin, Australia. We used 3D X-ray micro-CT imaging to characterise the pore structure of the rock and to obtain in-situ pore-scale images of the distribution of $\mathrm{CO}_{2}$ :brine analogue fluid pairs (octane:brine) during low capillary number drainage and imbibition displacements. The images showed that a significant portion of the non-wetting phase is rendered immobile in the pore space as disconnected clusters surrounded by brine.

The micro-CT images are used directly as input to a new network extraction and simulation workflow. We simulate primary drainage and imbibition displacements assuming quasi-static pore-scale physics. The calculated pore-scale fluid distributions are compared directly with the corresponding fluid distributions observed in the micro-CT flooding experiments. The simulated and experimental pore filling states are in good agreement. These results indicate that quasi-static assumptions can be used to obtain good predictions of average or continuum flow properties for water wet rocks at low capillary number displacements. Computed capillary trapping curves are in good agreement with the experimental results and confirm that residual trapping can be a key mechanism for $\mathrm{CO}_{2}$ storage. We carried out a sensitivity study of the impact wettability (i.e. advancing contact angle) on the magnitude of capillary trapping. As expected, the amount of $\mathrm{CO}_{2}$ trapping increases with decreasing contact angle.

\section{Acknowledgments}

The authors wish to acknowledge financial assistance provided through Australian National Low Emissions Coal Research and Development (ANLEC R\&D). ANLEC R\&D is supported by Australian Coal Association Low Emissions Technology Limited and the Australian Government through the Clean Energy Initiative.

\section{References}

Akbarabadi, M., Piri, M., 2013. Relative permeability hysteresis and capillary trapping characteristics of supercritical $\mathrm{CO}_{2}$ /brine systems: An experimental study at reservoir conditions. Adv. Water Resour. 52, 190-206.

Andrew, M., Bijeljic, B., Blunt, M., 2013. Pore-scale imaging of geological carbon dioxide storage under in-situ conditions. Geophys. Res. Lett. 40, 3915-3918.

Andrew, M., Bijeljic, B., Blunt, M., 2014a. Pore-scale contact angle measurement at reservoir conditions using X-ray microtomography. Adv. Water Resour. 68, 24-31.

Andrew, M., Bijeljic, B., Blunt, M., 2014b. Pore-scale imaging of trapped supercritical carbon dioxide in sandstones and carbonates. Int. J. Greenhouse Gas Control 22, $1-14$.

Berg, S., Ott, H., Klapp, S., Schwing, A., Neitler, R., Brusse, N., Makurat, A., Leu, L., Enzmann, F., Schwartz, J.O., Kersten, M., Irvine, S., Stampanoni, M., 2013. Real-time 
3D imaging of haines jumps in porous media flow. Proc. Natl. Acad. Sci. U.S.A. 110, 3755-3759.

Blunt, M., Bijeljic, B., Dong, H., Gharbi, O., Iglauer, S., Mostaghimi, P., Paluszny, A., Pentland, C., 2013. Pore-scale imaging and modelling. Adv. Water Resour. 51.

Blunt, M., King, J., Scher, H., 1992. Simulation and theory of two-phase flow in porous media. Phys. Rev. A 46.

Braun, E., Holland, R., 1995. Relative permeability hysteresis: laboratory measurements and a conceptual model. SPE Reservoir Eng. 10, 222-228.

Bultreys, T., Lin, Q., Gau, Y., Raeni, A., AlRatrout, A., Bijeljic, B., Blunt, M., 2018. Validation of model predictions of pore-scale fluid distributions during two-phase flow. Phys. Rev. E 97, 053104.

Burnside, N., Naylor, M., 2014. Review and implications of relative permeability of $\mathrm{CO}_{2}$ / brine systems and residual trapping of $\mathrm{CO}_{2}$. Int. J. Greenhouse Gas Control 23, 1-11. https://doi.org/10.1016/j.ijggc.2014.01.013.

Carlson, F., 1981. Simulation of Relative Permeability Hysteresis to the Nonwetting Phase. SPE-10157-MS.

Chatzis, I., Morrow, N., 1984. Correlation of capillary number relationships for sandstones. SPE J. 24, 555-562. https://doi.org/10.2118/10114-PA.

Dong, H., Blunt, M., 2009. Pore-network extraction from micro-computerized-tomography images. Phys. Rev. E 80, 036307.

Ferrari, A., Jimenez-Martinez, J., Borgne, T.L., Meheust, Y., Lunati, I., 2015. Challenges in modeling unstable two-phase flow experiments in porous micromodels. Water Resour. Res. 51, 1381-1400.

Herring, A., Andersson, L., Wildenschild, D., 2016. Enhancing residual trapping of supercritical $\mathrm{CO}_{2}$ via cyclic injections. Geophys. Res. Lett. 43, 9677-9685. https://doi. org/10.1002/2016GL070304.

Hilfer, R., Armstrong, R., Berg, S., Georgiadis, A., Ott, H., 2015. Capillary saturation and desaturation. Phys. Rev. E 92, 063023. https://doi.org/10.11103/PhysRevE.92. 063023.

Hilfer, R., Øren, P., 1996. Dimensional analysis of pore scale and field scale immiscible displacement. Transport Porous Media 22, 53-72. https://doi.org/10.1007/ BF00974311.

Khishvand, M., Akbarabadi, M., Piri, M., 2016. Micro-scale experimental investigation of the effect of flow rate on trapping in sandstone and carbonate rock samples. Adv. Water Resour. 94, 379-399. https://doi.org/10.1016/j.advwatres.2016.05.012.

Killough, J., 1976. Reservoir simulation with history-dependent saturation functions. SPE J. 16 (1), 37-48. https://doi.org/10.2118/5106-PA.

Kovscek, A., Radke, C., 1993. A pore-level scenario for the development of mixed wettability in oil reservoirs. AIChE J. 39, 1072-1085.

Krevor, S., Blunt, M., Benson, S., Pentland, C., Reynolds, C., Al-Menhali, A., Niu, B., 2015. Capillary trapping for geological carbon dioxide storage - from pore scale physics to field scale implications. Int. J. Greenhouse Gas Control 40, 221-237. https://doi.org/ 10.1016/j.ijggc.2015.04.006.

Krevor, S., Pini, R., Zuo, L., Benson, S., 2012. Relative permeability and trapping of $\mathrm{CO}_{2}$ and water in sandstone rocks at reservoir conditions. Water Resour. Res. 48. https:// doi.org/10.1029/2011WR010859.

Lake, L., 1989. Enhanced Oil Recovery. Prentice Hall, New York.

Land, C., 1968. Calculation of imbibition relative permeability for two-phase and threephase flow from rock properties. SPE J. 8, 149-156. https://doi.org/10.2118/ 1942-PA.

Lee, T., Kashyap, R., Chu, C., 1994. Building skeleton models via 3-d medial suface axis thinning algorithms. Graphical Models Image Process. 56, 462-478.

Lenormand, R., Zarcone, C., Sarr, A., 1983. Mechanisms of displacement of one fluid by another in a network of capillary ducts. J. Fluid Mech. 135, 337-353.

Ling, B., Bao, J., Oostrom, M., Battiato, I., Tartakovsky, A., 2017. Modeling variability in porescale multiphase flow. Adv. Water Resour. 105, 29-38.

Mayer, R., Stowe, R., 1965. Mercury porosimetry-breakthrough pressure for penetration between packed spheres. J. Colloid Interface Sci. 20, 893-911.

Menke, H., Andrew, M., Blunt, M., Bijeljic, B., 2016. Reservoir condition imaging of reactive transport in heterogenous carbonates using fast synchrotron tomography - effect of initial pore structure and flow conditions. Chem. Geol. 428, 15-25.

Niu, B., Al-Menhali, A., Krevor, S., 2015. The impact of reservoir conditions on the residual trapping of carbon dioxide in Berea sandstone. Water Resour. Res. 51, 2009-2029. https://doi.org/10.1002/2014WR016441.

Øren, P., Bakke, S., 2003. Reconstruction of Berea sandstone and pore-scale modelling of wettability effects. J. Petrol. Sci. Eng. 39, 177-199.

Øren, P., Bakke, S., Arntzen, O., 1998. Extending predictive capabilities to network models. SPE J. 3, 324-336.

Pentland, C., El-Maghraby, R., Iglauer, S., Blunt, M., 2011. Measurements of the capillary trapping of super-critical carbon dioxide in Berea sandstone. Geophys. Res. Lett. 38.

Piri, M., Blunt, M., 2004. Three-phase threshold capillary pressures in noncircular capillary tubes with different wettabilities including contact angle hysteresis. Phys. Rev. E 70.

Princen, H., 1969. Capillary phenomena in assemblies of parallel cylinders. i. Capillary rise between two cylinders. J. Colloid Interface Sci. 30, 69-75.

Raeini, A., Bijeljic, B., Blunt, M., 2015. Modelling capillary trapping using finite-volume simulation of two-phase flow directly on micro-CT images. Adv. Water Resour. 83, $102-110$.

Ruspini, L., Farokhpoor, R., Øren, P., 2017. Pore-scale modeling of capillary trapping in water-wet porous media: a new cooperative pore-body filling model. Adv. Water Resour. 108, 1-14.

Saito, T., Torimaki, J.I., 1994. New algorithms for Euclidean distance transformation of an $n$-dimensional digitized picture with applications. Pattern Recognit. 27, 1551-1565.

Schlüter, S., Berg, S., Li, T., Vogel, H.J., D.W, 2017. Time scales of relaxation dynamics during transient conditions in two-phase flow. Water Resour. Res. 53, 4709-4724.

Sheppard, A., Sok, R., Averdunk, H., 2004. Technique for image enhancement and segmentation of tomographic images of porous materials. Physica A 339, 145-151.

Silin, D., Patzek, T., 2003. Robust Determination of the Pore Space Morphology in Sedimentary Rocks. https://doi.org/10.2118/84296-MS. SPE 84296-MS.

Song, W., Kovscek, A., 2016. Direct visualization of pore-scale fines migration and formation damage during low-salinity waterflooding. J. Nat. Gas Sci. Eng. 34, 1276-1283.

Spiteri, E., Juanes, R., Blunt, M., Orr, F., 2008. A new model of trapping and relative permeability hysteresis for all wettability characteristics. SPE J. 277-288.

Suzanne, K., Hamon, G., Trocme, V., 2003. Experimental Relationships between Residual Gas Saturation and Initial Gas Saturation in Heterogeneous Sandstone Reservoirs. SPE 84038.

Taber, J., 1969. Dynamic and static forces required to remove a discontinuous oil phase from porous media containing both oil and water. SPE J. 9, 3-12. https://doi.org/10. 2118/2098-PA.

van Dijke, M., Lago, M., Sorbie, K., Araujo, M., 2004. Free energy balance for three fluid phases in a capillary of arbitrarily shaped cross-section: capillary entry pressures and layers of the intermediate-wetting phase. J. Colloid Interface Sci. 277, 184-201.

van Dijke, M., Piri, M., Helland, J., Sorbie, K., Blunt, M., 2007. Criteria for three-phase configurations including layers in a pore with nonuniform wettability. Water Resour. Res. 43, 1-11.

van Dijke, M., Sorbie, K., 2006. Existence of fluid layers in the corners of a capillary with nonuniform wettability. J. Colloid Interface Sci. 293, 455-463.

Wildenshild, D., Sheppard, A., 2013. X-ray imaging and analysis techniques for quantifying pore-scale structure and processes in subsurface porous medium systems. Adv. Water Resour. 51, 217-246.

Yi, Z., Lin, M., Jiang, W., Zhang, Z., Li, H., Gao, J., 2017. Pore network extraction from pore space images of various porous media systems. Water Resour. Res. 53, 3424-3445.

Youssef, S., Peysson, Y., Bauer, D., Vizika, O., 2015. Capillary desaturation curve prediction using 3D microtomography images. In: International Symposium of the Society of Core Analysis. St John's, Newfoundland, Canada.

Zuo, L., Benson, S., 2014. Process-dependent residual trapping of $\mathrm{CO}_{2}$ in sandstone. Geophys. Res. Lett. 41, 2820-2826. https://doi.org/10.1002/2014GL059653. 